Physica 106B (1981) 59-67

North-Holland Publishing Company

\title{
FLUX PENETRATION AND a.c. LOSSES IN TYPE II SUPERCONDUCTORS
}

\author{
I. MODEL CALCULATIONS \\ E. A. GIJSBERTSE, J. SIKKENGA and L. J. M. VAN DE KLUNDERT \\ Department of Applied Physics, Twente University of Technology, P.O. Box 217, 7500 AE Enschede, The Netherlands
}

Received 22 December 1980

\begin{abstract}
A numerical method is presented which allows the calculation of the a.c. response of type ll superconductors under quasi-static conditions for arbitrary $j_{c}(B, x), B_{\text {en }}\left(B_{a}\right)$ and $B_{\mathrm{ex}}\left(B_{\mathrm{a}}\right)$ relations. Flux distributions, induced voltage waveforms, hysteresis loops and a.c. losses have been calculated. Deviations between experimental results and earlier calculations with respect to a minimum in the a.c. losses are explained by taking into account the finite thickness of the sample and realistic $B_{\mathrm{en}}\left(B_{\mathrm{a}}\right)$ and $B_{\mathrm{ex}}\left(B_{\mathrm{a}}\right)$ relations.
\end{abstract}

\section{Introduction}

A generally accepted model for the phenomenological description of the behaviour of type II superconductors is the Critical State Model (CSM) [1] . In this model the superconducting bulk currents are described in terms of a critical current density $j_{c}$. Reversible surface screening currents can be denoted by a $B_{\text {eq }}\left(B_{\mathrm{a}}\right)$ relation which in the irreversible case is replaced by $B_{\text {en }}\left(B_{\mathrm{a}}\right)$ for an increasing external field $B_{\mathrm{a}}$ and by $B_{\mathrm{ex}}\left(B_{\mathrm{a}}\right)$ in decreasing fields. Under a.c. conditions over-critical currents may also occur which can be described in the same way as normal currents in terms of a flux flow conductivity $\sigma_{\mathrm{ff}}$. A more detailed description of this isothermal CSM has been given elsewhere $[2-4]$. In the literature a large number of model calculations, mostly concerning a.c. losses, has been presented. In many cases these calculations have been restricted to simplified models and one-dimensional geometries which allow analytical solutions. Surprisingly, only a few attempts to solve the appropriate equations numerically, have been made. Numerical methods allow the calculation of flux distributions, induced voltage waveforms, hysteresis loops and a.c. losses in many situations. The various parameters of the CSM may assume any arbitrary dependence on magnetic induction, position etc. and also the waveform of the applied magnetic field and the geometry of the sample may be varied. Although in this case simple models are preferred too, due to limitations of computer usage, a numerical approach may expand the range in which solutions can be obtained, considerably.

Quasi-static calculations have first been presented by Walmsley [5] taking into account several $j_{\mathrm{c}}(B)$ relations. Irreversible surface currents have been neglected but reversible screening currents have been included in the model in terms of a local $B_{\text {eq }}(H)$ relation. Clem [6] has developed a computer program which allows the calculation of flux distributions, induced voltage waveforms, hysteresis loops and a.c. loss values for various pinning models in linearly varying a.c. fields. In addition to Walmsley's model, surface irreversibilities have been included in terms of a $B_{\text {en }}\left(B_{\mathrm{a}}\right)$ and a $B_{\text {ex }}\left(B_{\mathrm{a}}\right)$ relation. Here, however, the calculations have been restricted to small depths of penetration which means that the influence of the finite dimensions of the sample has not been considered. Clem's calculations have led to the prediction of a minimum of the a.c. losses when a static field $B_{0}$ is superposed on the a.c. field. Although the occurrence of such a minimum has indeed been observed in a number of materials [7-9], the experimental results do not agree with Clem's calculations on various aspects [9].

In this paper a further extension of these quasi- 
static model calculations will be presented in which both the finite dimensions of the sample and the irreversibilities of the surface currents have been included. The consequences of an explicit dependence of $j_{c}$ on position have also been considered. The calculations have been performed for a slab geometry. In contrast to the models of Walmsley and Clem, a $B_{\text {eq }}(H)$ relation will not be used. Instead, an alternative formulation of the CSM will be employed in which all superconducting bulk currents are incorporated in $j_{c}$ [4]. The numerical techniques will be discussed in section 2. After that some calculated induced voltage curves and hysteresis loops will be presented in section 3 . In section 4 calculated results with respect to the minimum in a.c. losses will be given and compared with the experimental results followed by a discussion in section 5 .

For a comparison of calculated and experimental hysteresis loops we refer to a separate publication (part II) [10]. The calculations have also been used to verify an experimental technique which has been proposed by the authors. This technique has been presented elsewhere together with the numerical verification $[3,4]$.

\section{Numerical techniques}

In quasi-static model calculations, the flux diffusion equation (in slab geometry) reads:

$$
\partial B / \partial x= \pm \mu_{0} j_{c}(B, x),
$$

where the sign depends on the position inside the sample. The position $x_{t}$ at which the change of sign occurs (the flux front) is determined by the value of the applied field $B_{\mathrm{a}}$ and the value of $B_{\mathrm{a}}$ at which the variation of the external field started. (1) can be solved for any $B_{\mathrm{a}}$ value at every position in a onedimensional, equidistant array of $x$-values. For numerical convenience, the values of $B_{\mathrm{a}}(t)$ have been chosen in such a way that the flux front $x_{t}$ always coincides with the points of this grid. This choice implies a non-equidistant $B_{\mathrm{a}}$ array.

The numerical calculations may be reduced considerably when the critical current density is assumed to depend on magnetic induction only. In this case all flux distributions may be derived from a universal flux profile function $B_{\text {un }}\left(x^{\prime}\right)$ :

$B_{\text {un }}\left(x^{\prime}\right)=\mu_{0} \int_{0}^{x^{\prime}} j_{c}(B(x)) \mathrm{d} x$.

This method has previously been used by Walmsley [5]. Solutions for arbitrary boundary-conditions can be obtained by shifting the slab along this universal curve until the value at the surface satisfies the boundary condition. As a consequence of the symmetry of the flux distribution with respect to the flux front, both branches of the flux profile can be determined from the same $B_{\text {un }}$ curve. A problem may arise when the external field is swept around zero. However, due to the definition of $B_{\text {un }}$ (see eq. (2)) the zero-crossing of the flux distribution also coincides with the points of the $x$-array and the anti-symmetry of the flux profile function with respect to $B=0$ can be used to avoid extra calculation. In fig. 1 some calculated flux distributions in an applied a.c. field of amplitude $b_{0}$ superposed on a static field $B_{0}$ are shown. The shape of the flux distribution is independent of the waveform of the applied field. Also the presence of surface screening currents leaves the profiles unaffected; these currents only influence the value of the external field at which the flux distribution occurs.

The values of the average induction $B_{\mathrm{av}}$ inside the sample can be derived from a second universal curve: the total flux function $\phi_{u n}\left(x^{\prime}\right)$ which is the primitive function of $B_{\mathrm{un}}\left(x^{\prime}\right)$. With the help of the $B_{\mathrm{av}}$ values, the hysteresis loop and the a.c. loss per cycle can be calculated. It may be noted that under quasi-static conditions the shape of the hysteresis loop is independent of the applied field waveform. The time dependence of the applied field only affects the induced voltage waveform which can be obtained by differentiation of the average induction with respect to time. In this paper only linear variations of $B_{\mathrm{a}}(t)$ will be considered in accordance with the trapezoidal waveform in the experiments.

When an explicit position dependence of $j_{c}$ is included in the model, the universal function method cannot be used any longer. In this case both the flux distribution and the average induction have to be calculated for each individual $B_{\mathrm{a}}$ value by numerical integration of eq. (1). The results obtained in this way indicate that no substantial deviations follow 


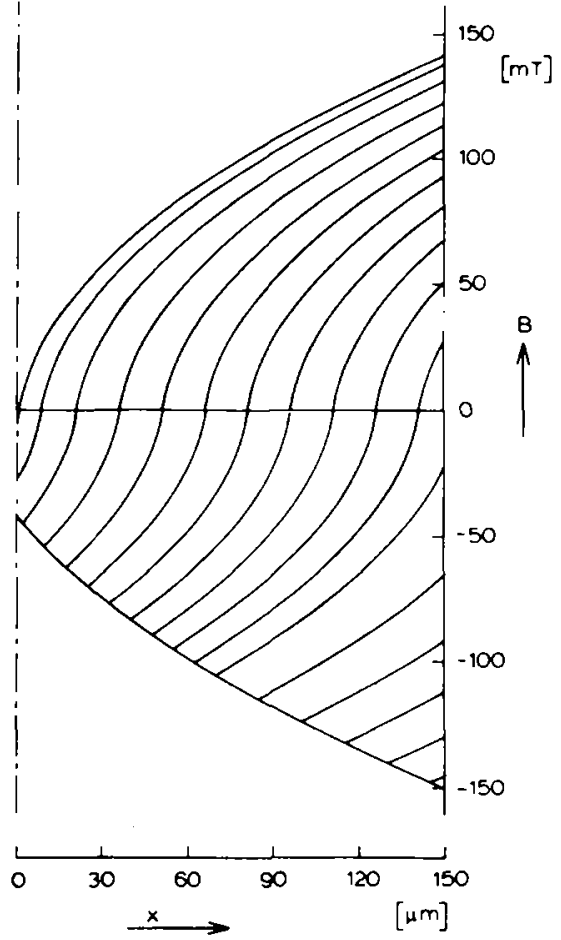

Fig. 1. Calculated flux density distributions during the increasing half of the a.c. field $\left(B_{0}=0 \mathrm{mT} ; b_{0}=150 \mathrm{mT}\right)$ for a slab with thickness $2 d=0.30 \mathrm{~mm}$. The critical current density $j_{\mathrm{c}}$ depends on $B$ according to eq. (3); $\left(j_{\mathrm{c}}(0)=0.637\right.$ $\times 10^{6} \mathrm{~A} \mathrm{~cm}^{-2} ; B_{1}=10 \mathrm{mT}$; no surface currents).

from an inclusion of an explicit $j_{\mathrm{c}}(x)$ relation. When the $x$-dependence is restricted to a narrow layer near the surface, the response strongly resembles the effect of surface screening. Otherwise, the results are analogous to those obtained in the case of uniform bulk pinning. For this reason no calculations involving a position dependent $j_{c}$ will be presented here.

\section{Induced voltage waveforms and hysteresis loops}

Numerical calculations have beèn performed on a slab of thickness $2 d=0.30 \mathrm{~mm}$, in accordance with the thickness of the slabs used in the experiments. A Kim-like relation [11] has been used for the $B$. dependence of $j_{\mathrm{c}}$ :

$j_{\mathrm{c}}(B)=j_{\mathrm{c}}(0) /\left(1+|B| / B_{1}\right)$.

The parameters $j_{\mathrm{c}}(0)$ and $B_{1}$ have been chosen in agreement with the values obtained from experiments on a $\mathrm{Nb}-50 \% \mathrm{Ti}$ slab [10]. The influence of the variation of these parameters has also been studied. In the case of eq. (3) the two universal functions $B_{\text {un }}$ and $\phi_{\text {un }}$ can be integrated analytically.

In fig. 2 some calculated induced voltage waveforms for a slab without surface screening currents in a linearly increasing magnetic field and various values of the static field $B_{0}$ have been shown. The $E$-values have been normalized with $\alpha d$, the value of $E$ for a non-magnetic non-conducting material $\left(\alpha=\mathrm{d} B_{\mathrm{a}} / \mathrm{d} t\right)$. When the external field $B_{\mathrm{a}}$ is raised from a very low value, the envelope curve decreases from its original value 1 and goes through a minimum in the vicinity of $B_{\mathrm{a}}=0$. After that a sharp increase can be observed leading to a maximum and subsequently a decay to $E / \alpha d=1$ for high positive fields. A similar behaviour has been observed in the case of a normal metal with a $B$-dependent conductivity $[4,12]$. This behaviour can be understood in terms of a simple qualitative model. When a type II superconductor with a $j_{\mathrm{c}}$ linear in $B$, i.e.

$j_{\mathrm{c}}(B)=j_{0}(1+\beta B)$,

is placed in a linearly increasing magnetic field, a simple analytical calculation leads to the following expression for the envelope curve:

$E / \alpha d=\frac{1}{\beta^{\prime}}\left(1-\mathrm{e}^{-\beta^{\prime}}\right) \approx 1-\frac{1}{2} \beta^{\prime}$,

where $\beta^{\prime}=\mu_{0} j_{0} \beta d=\mu_{0} d \partial j_{\mathrm{c}} / \partial B$. In the high field

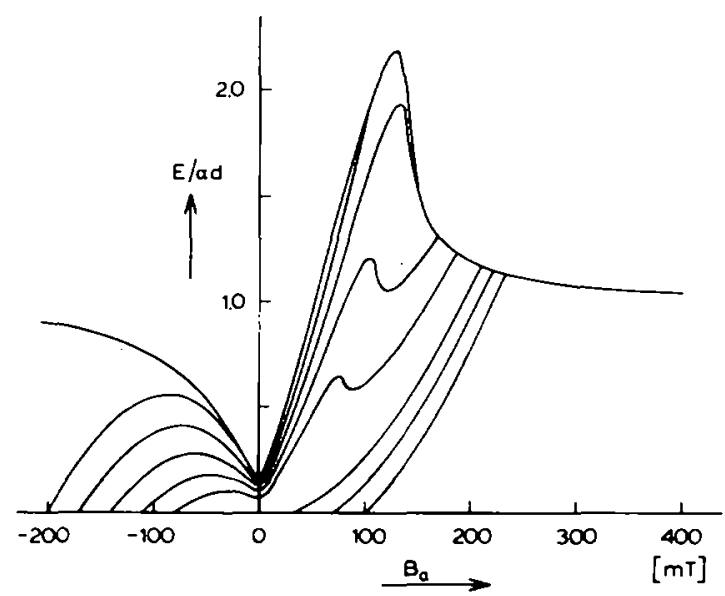

Fig. 2. Induced voltage waveforms for various values of the static field $B_{0}$ (see fig. 1); no surface currents. 
region the first order Taylor expansion shown in eq. (4) is a good approximation of the Kim relation (3). In the derivation of eq. (5) the property that at the envelope curve the flux front has already reached the centre plane, has been used. The approximation in eq. (5) is only valid for small values of $\beta^{\prime}$. It can be seen from eq. (5) that for positive values of $\partial j_{c} / \partial B$, the value of $E / \alpha d$ is smaller than 1 and vice versa. The sharp increase of the envelope curve in the vicinity of $B_{\mathrm{a}}=0$ can be explained by a change of sign of $\partial j_{\mathrm{c}} / \partial B$. It can be understood from the model that the peak in $E$ shifts to the left when the critical current density (i.e. $\left.j_{\mathrm{c}}(0)\right)$ is decreased. In this case the average induction $B_{\mathrm{av}}$ goes to zero at a higher value of $B_{\mathrm{a}}$. A decrease of the constant $B_{1}$ results in a higher value of $\partial j_{c} / \partial B$ and therefore in a sharper peak. Both points have been confirmed by the numerical calculations. Although the shape of the envelope curve can be understood qualitatively from the arguments given above, it is not possible to determine the $j_{c}(B)$ relation directly from the envelope curve. This is a consequence of the variation of $j_{\mathrm{c}}$ inside the sample leading to a complex relationship between $j_{\mathrm{c}}$ and the envelope curve. As expected the individual $E / \alpha d$ curves have a sharp edge when they reach the envelope curve.

The inclusion of surface screening currents in the model in terms of $B_{\mathrm{en}}\left(B_{\mathrm{a}}\right)$ and a $B_{\mathrm{ex}}\left(B_{\mathrm{a}}\right)$ relation does not affect the shape of the flux distribution but only the external field at which this distribution occurs. The surface screening currents have been assumed to depend on $B_{\mathrm{a}}$ in the following way:

$\begin{array}{ll}B_{\text {en }}\left(B_{\mathrm{a}}\right)=B_{\mathrm{c}_{2}} \frac{\sqrt{\left(B_{\mathrm{a}}^{2}-B_{\mathrm{s}}^{2}\right)}}{\sqrt{\left(B_{\mathrm{c}_{2}}^{2}-B_{\mathrm{s}}^{2}\right)}} ; & B_{\mathrm{s}} \leqslant B_{\mathrm{a}} \leqslant B_{\mathrm{c}_{2}} \\ B_{\text {en }}\left(B_{\mathrm{a}}\right)=0 ; & 0 \leqslant B_{\mathrm{a}} \leqslant B_{\mathrm{s}} ;\end{array}$

$B_{\mathrm{ex}}\left(B_{\mathrm{a}}\right)=B_{\mathrm{a}}$.

Eq. (6) is a variation of the theoretical result of Clem [13] for a high $\kappa$ superconductor which also takes the value of the second critical field into account. $B_{\mathrm{s}}$ is the superheating field. The calculations have resulted in induced voltage waveforms with a zero value of $E$ in the interval $0 \leqslant B_{\mathrm{a}} \leqslant B_{\mathrm{s}}$. This corresponds to an interval with constant average induction in the hysteresis loops. This interval is not shown in fig. 3 where the values of the $E$-curves at $B_{\mathrm{a}}=0$ and $B_{\mathrm{a}}=B_{\mathrm{s}}$ have been connected by straight lines. This arbitrary connection function (a consequence of the graphical computer technique) has not been replaced by an interval with $E=0$ because such an interval has never been observed experimentally. This may be attributed to inhomogeneous surface screening [14]. Some examples of calculated induced voltage waveforms and hysteresis loops are shown in figs. 3 and 4. A

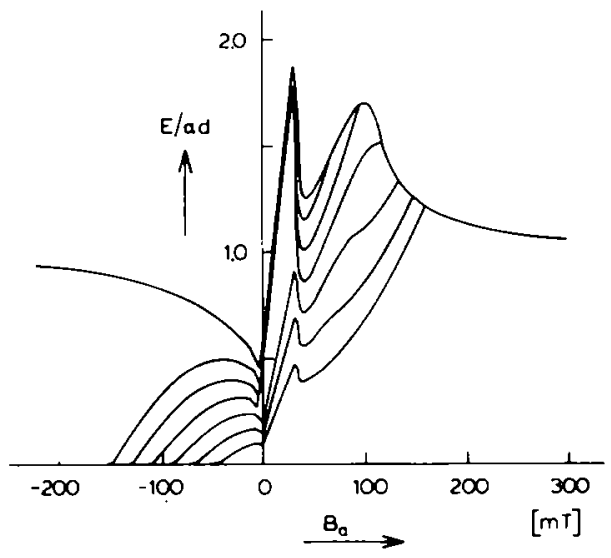

Fig. 3. Induced voltage waveforms for various values of the static field $B_{0}$; surface screening currents have been included according to eqs. (6) and (7). In the region $0<B_{\mathrm{a}}<B_{\mathrm{g}}$ an arbitrary connection function has been used $\left(b_{0}=150 \mathrm{mT}\right.$; $\left.j_{\mathrm{c}}(0)=0.127 \times 10^{6} \mathrm{~A} \mathrm{~cm}^{-2} ; B_{1}=50 \mathrm{mT} ; B_{\mathrm{s}}=30 \mathrm{mT}\right)$.

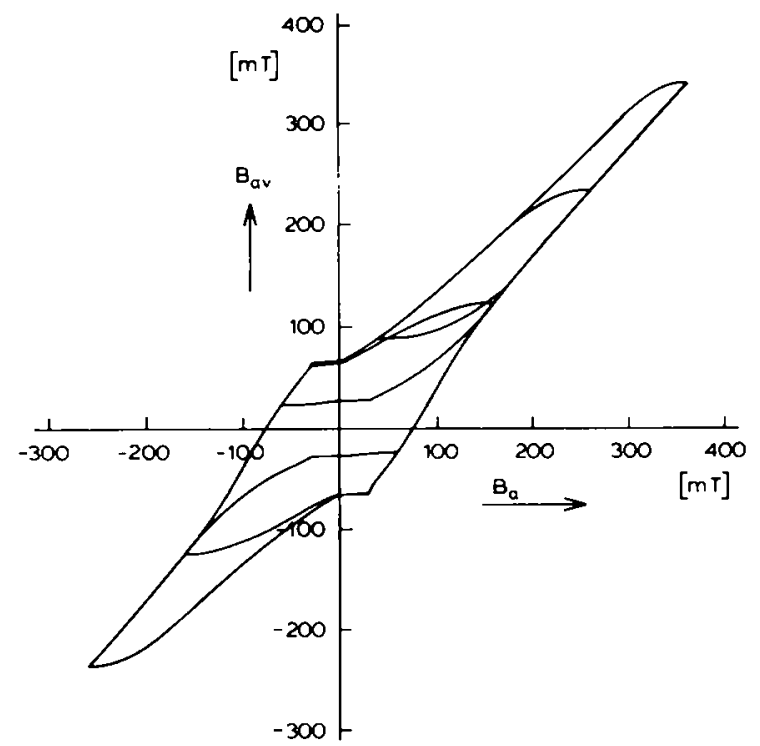

Fig. 4. Hysteresis loops for various values of the static field $B_{0}$ (see fig. 3). 
remarkable feature of the induced voltage waveforms is the occurrence of a double peak in the envelope curve; one peak is related to bulk currents, the other to surface currents.

In this paper only calculations involving the theoretical $B_{\text {en }}\left(B_{\mathrm{a}}\right)$ and $B_{\text {ex }}\left(B_{\mathrm{a}}\right)$ relations (6) and (7) are presented. The results from calculations with experimental $B_{\text {en }}$ and $B_{\text {ex }}$ curves will be presented in part II with the experimental induced voltage waveforms. Only some results concerning the minimum in a.c. losses will be presented in section 4 .

\section{A minimum in a.c. losses}

\subsection{Experimental situation}

Numerical calculations by Clem [6] have led to the conclusion that the a.c. losses in type II superconductors may exhibit a minimum when a d.c. magnetic field $B_{0}$ is superposed to an a.c. field with amplitude $b_{0}$. The occurrence of such a minimum can be explained qualitatively by the CSM provided that a magnetic field dependence of the critical current density $j_{\mathrm{c}}$ and/or $B_{\mathrm{en}}$ and $B_{\mathrm{ex}}$ is taken into account. Clem's calculations predict a minimum at an external field $B_{\text {min }}=b_{0}-B_{\text {en }}(0)$ with $b_{0}>B_{\text {en }}(0)$ when the $B$-dependence of the critical entry field is considered and a minimum at $B_{\min } \approx \frac{2}{3} b_{0}$ in the case of $j_{\mathrm{c}}(B)$ according to eq. (3).

Recent experiments have shown the existence of such a minimum in various materials such as $\mathrm{Nb}_{3} \mathrm{Ge}$ [7] , $\mathrm{Nb}-10 \% \mathrm{Mo}$ [8] and $\mathrm{Nb}-50 \% \mathrm{Ti}$ [9]. The results for the two latter materials have been summarized in fig. 5 where the external field $B_{\min }$ at which the minimum occurs has been plotted vs. $b_{0}$. Two deviations with respect to Clem's predictions can be observed [9] .

(1) The slopes of the straight lines which can be drawn through the experimental points are not in agreement with the values 1 and $\frac{2}{3}$ calculated by Clem. Only at large amplitudes $\left(b_{0}>45 \mathrm{mT}\right)$ do the results for $\mathrm{Nb}-50 \% \mathrm{Ti}$ fit the theoretical result reasonably in the case of a $\mathrm{Kim}$ relation for $j_{\mathrm{c}}(B)$ (the slope $n=0.69$ ). The slope at smaller amplitudes ( $n=0.86$ ) suggests a combination of surface and bulk currents. A similar result has been observed in $\mathrm{Nb}_{3} \mathrm{Ge}$ : $B_{\text {min }}=0.92 b_{0}-81.4 \mathrm{mT}$ [7] . for $\mathrm{Nb}-10 \% \mathrm{Mo} \mathrm{a}$ slope $n=1.40$ has been observed.

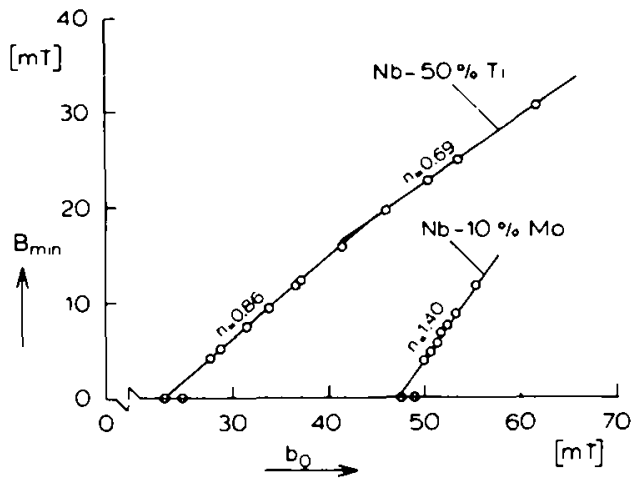

Fig. 5. The static field at which the minimum in a.c. losses occurs as a function of amplitude for $\mathrm{Nb}-50 \% \mathrm{Ti}$ and $\mathrm{Nb}-10 \% \mathrm{Mo}$ (experimental).

(2) According to Clem the lines through the experimental points should cross the line $B_{\min }=0$ at an amplitude $b_{0}$ equal to the field of first penetration $B_{\mathrm{s}}$. However, for $\mathrm{Nb}-50 \% \mathrm{Ti}$ this value of $b_{0}$ $\left(b_{0} \approx 23 \mathrm{mT}\right)$ is larger than the one measured directly by looking at the induced voltage waveform $\left(b_{0}\right.$ $=14 \mathrm{mT}$ ). Even at amplitudes slightly exceeding the point of intersection $B_{\min }$ is still zero as can be seen in fig. 5 for both $\mathrm{Nb}-50 \% \mathrm{Ti}$ and $\mathrm{Nb}-10 \% \mathrm{Mo}$. The same feature can be observed in the results presented by Thompson et al. [7] for $\mathrm{Nb}_{3} \mathrm{Ge}$ where $B_{\text {min }}=0$ at an amplitude $b_{0}=125 \mathrm{mT}$ while the point of intersection lies at $b_{0}=88.5 \mathrm{mT}$.

These deviations may possibly be avoided by making some additions to Clem's model. In the first place the influence of the finite thickness of the slab will be taken into account. Secondly both bulk and surface currents will be considered. Finally the influence of more realistic $B_{\mathrm{en}}\left(B_{\mathrm{a}}\right)$ and $B_{\mathrm{ex}}\left(B_{\mathrm{a}}\right)$ relations will be studied.

\subsection{Finite thickness of the slab}

In order to study the influence of the finite thickness of the sample, model calculations have been performed for a large number of amplitudes and static fields. The a.c. losses have been calculated in the case of bulk currents only. The critical current density $j_{\mathrm{c}}$ is given by relation (3): $j_{c}(0)$ and $B_{1}$ have been taken in reasonable agreement with the experimental values for $\mathrm{Nb}-50 \% \mathrm{Ti}[10]$. In fig. 6 the a.c. losses have been plotted vs. $B_{0}$ for a large number of amplitudes. 


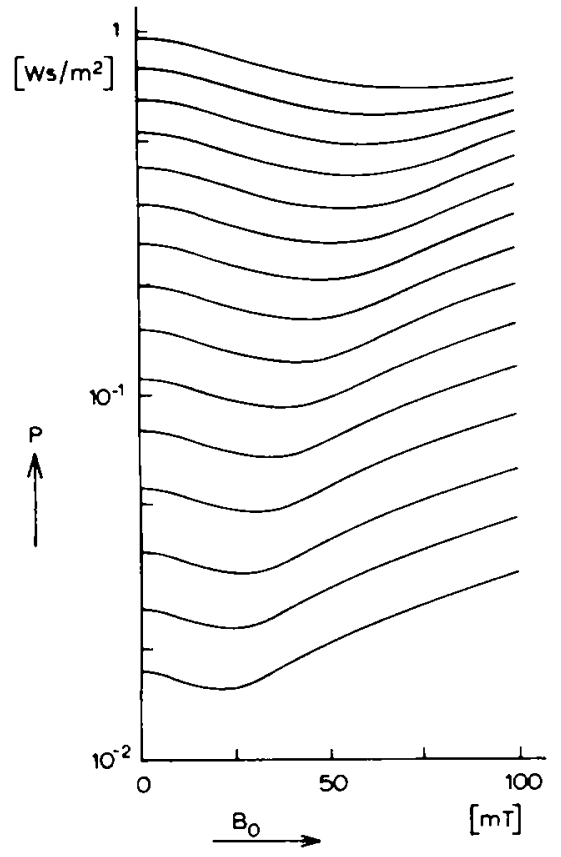

Fig. 6. Calculated a.c. losses as a function of static field for various amplitudes $\left(b_{0}=32,36,40,45, \ldots\right.$ step $5, \ldots$, $100 \mathrm{mT})$ with bulk currents only $\left(j_{\mathrm{c}}(0)=0.127 \times 10^{6} \mathrm{~A}\right.$ $\mathrm{cm}^{-2} ; B_{1}=50 \mathrm{mT}$ ).

It can be seen that a minimum occurs for every amplitude. The shape of the curves in the vicinity of the minimum is very smooth. The position of the minimum has been plotted vs. $b_{0}$ in fig. 7 . At small amplitudes the results agree with Clem's calculations:

$B_{\min }=\frac{2}{3} b_{0}$. However, when the flux front reaches the central plane of the slab during the a.c. cycle even at $B_{0}=0$, this relation is no longer valid. In the case presented here, a slope $n=1.2$ is found; other calculations have shown that in this part of the curve the slope depends on the parameters $i_{\mathrm{c}}(0)$ and $B_{1}$. These results show that slopes $n>1$ may be explained by taking into account the finite thickness of the sample. This influence may be understood from the fact that the a.c. losses at large amplitudes are smaller in a finite slab than in a semi-infinite space. From the foregoing it may be concluded that the slope $n=1.4$ observed for $\mathrm{Nb}-10 \% \mathrm{Mo}$ is a consequence of the influence of the finite dimensions of the sample; the occurrence of this slope immediately after appearance of a minimum may be attributed to the very small value of $j_{\mathrm{c}}$ in this sample. It has not been possible to

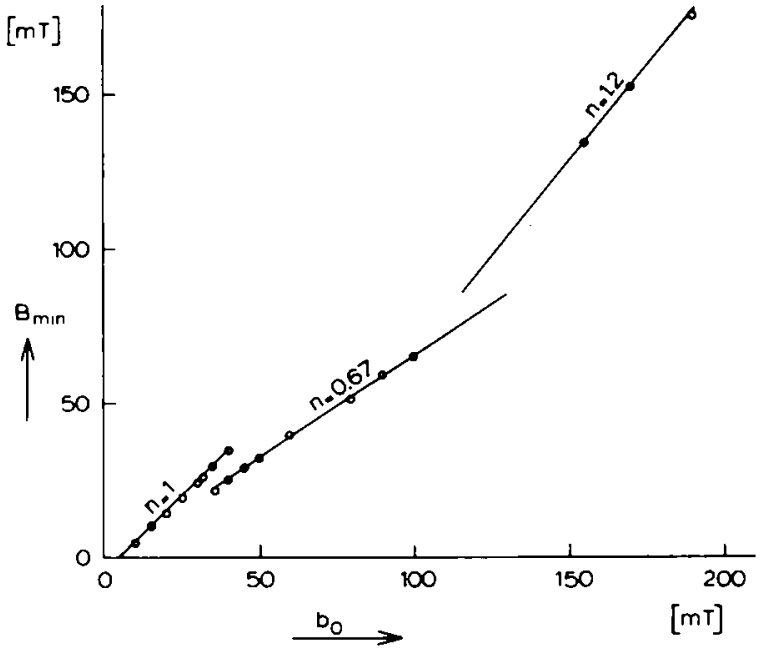

Fig. 7. The static field at which the minimum occurs as a function of amplitude without surface currents ( $n=0.67$ and $n=1.2$; see fig. 6 ) and with surface currents $(n=1$; see fig. 8).

verify this result by experiments on $\mathrm{Nb}-50 \% \mathrm{Ti}$ due to the large amplitude needed for these measurements.

\subsection{Contribution of bulk and surface currents}

A plot of the a.c. losses vs. $B_{0}$ in the case where surface currents are taken into account as well, is given in fig. 8. In these calculations the relations (6) and (7) have been assumed to describe the surface screening currents. The bulk currents have not been altered compared with fig. 6 . For small amplitudes the a.c. losses are much smaller than in the case with bulk currents only. In the plot of $B_{\min }$ vs. $B_{0}$ (see fig. 7) a straight line with slope $n=1$ is obtained which is in agreement with Clem's result; no variations of the slope have been observed when the central plane of the slab has been reached. The point of intersection with the line $B_{\min }=0$ is given by $B_{\mathrm{s}}$, the field of first penetration. As can be seen from fig. 8 the shape of the minimum due to surface currents is very sharp in contrast to the smooth minimum due to bulk currents. When both surface and bulk currents are present, the minimum related to the surface currents dominates in the low field region because these currents depend much more strongly on $B$ than the critical current density in this field range. 


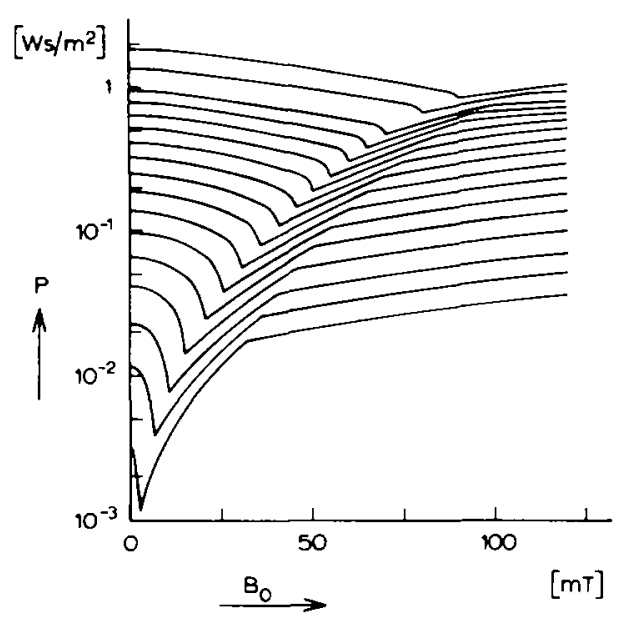

Fig. 8. Calculated a.c. losses as a function of static field for various amplitudes $\left(b_{0}=32,36,40,45, \ldots\right.$, step $5, \ldots, 95$, $100,110,120 \mathrm{mT})$ with bulk and surface currents $\left(j_{\mathrm{c}}(0)\right.$ $=0.127 \times 10^{6} \mathrm{~A} \mathrm{~cm}^{-2} ; B_{1}=50 \mathrm{mT} ; B_{\mathrm{s}}=30 \mathrm{mT}$ ).

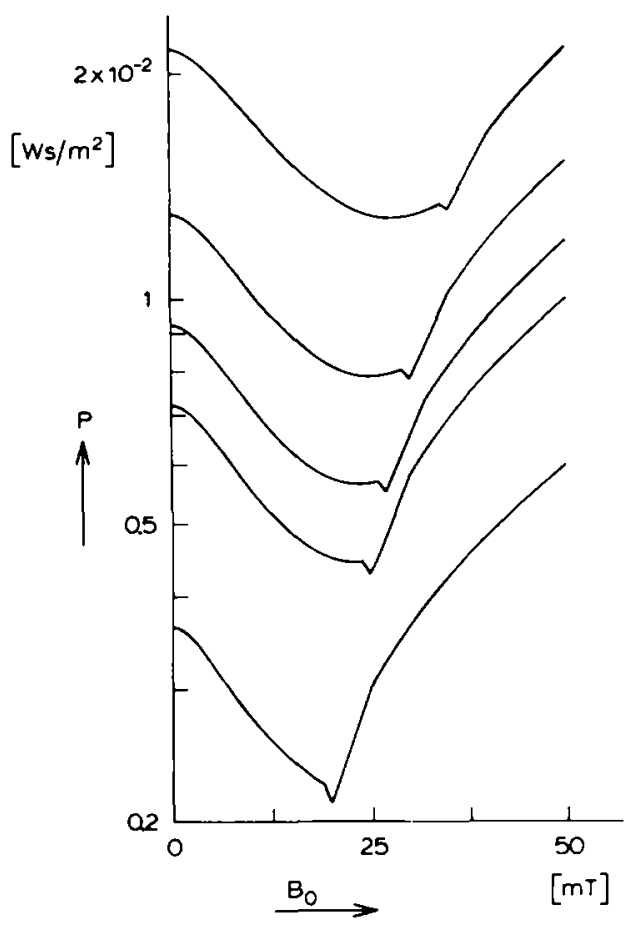

Fig. 9. Calculated a.c. losses vs. static field curves exhibiting a double minimum $\left(j_{\mathrm{c}}(0)=0.637 \times 10^{6} \mathrm{~A} \mathrm{~cm}^{-2} ; B_{1}\right.$ $\left.=10 \mathrm{mT} ; B_{\mathrm{s}}=5 \mathrm{mT}\right)$ for various amplitudes $\left(b_{0}=25,30,32\right.$, $35,40 \mathrm{mT}$ ).
A very interesting case arises when both contributions are comparable. In this case two minima can be distinguished: a sharp and a smooth one, related to surface and bulk currents respectively. Some results obtained in this case have been plotted in fig. 9. The positions of these two minima depend in their own characteristic way on $b_{0}$.

\subsection{Experimental relations for $B_{\mathrm{en}}\left(B_{\mathrm{a}}\right)$ and $B_{\mathrm{ex}}\left(B_{\mathrm{a}}\right)$}

In the preceding subsection the influence on the a.c. losses of surface screening currents described by the theoretical $B_{\text {en }}\left(B_{\mathrm{a}}\right)$ and $B_{\text {ex }}\left(B_{\mathrm{a}}\right)$ relations (6) and (7) has been studied. Experiments, however, have shown that these theoretical relations do not describe the behaviour of the $\mathrm{Nb}-50 \% \mathrm{Ti}$ slab properly. In the following some experimental $B_{\mathrm{en}}$ and $B_{\mathrm{ex}}$ curves will be introduced in the calculations. These curves have been determined with the help of trapezoidal a.c. fields $[3,4]$; the currents are in good agreement with results obtained with other techniques $[3,4,10$, 14]. The deviations between the experimental and theoretical curves can be characterized as follows:

(1) the $B_{\text {ex }}\left(B_{\mathrm{a}}\right)$ curve cannot be described by eq. (7) but has approximately the same functional dependence on $B_{\mathrm{a}}$ as $B_{\mathrm{en}}$;

(2) both $B_{\mathrm{en}}\left(B_{\mathrm{a}}\right)$ and $B_{\mathrm{ex}}\left(B_{\mathrm{a}}\right)$ vary continuously, i.e. there is no interval during which $B_{\mathrm{en}}$ or $B_{\mathrm{ex}}$ remains constant. This may be attributed to inhomogeneous surface screening [14].

The $B_{\mathrm{en}}$ and $B_{\mathrm{ex}}$ curves have been introduced in the computer program in the form of two arrays of experimental values; intermediate points have been determined by means of numerical interpolation. The critical current density has again be assumed to have a Kim-like dependence on magnetic induction.

A plot of the a.c. losses vs. static field has been given in fig. 10 for various amplitudes. Due to the smooth variation of the surface screening currents, no sharp minimum can be observed. The position of the minimum has been plotted vs. $b_{0}$ in fig. 11 . This figure shows a remarkable resemblance with the experimental results for the same sample (see fig. 7). The points of agreement may be summarized as:

(1) for high amplitudes the slope in the $B_{\min }$ vs. $b_{0}$ curve is $n=\frac{2}{3}$ which may be attributed to bulk currents. For smaller amplitudes a transition to a higher slope $(n=1)$ can be observed. This value, 


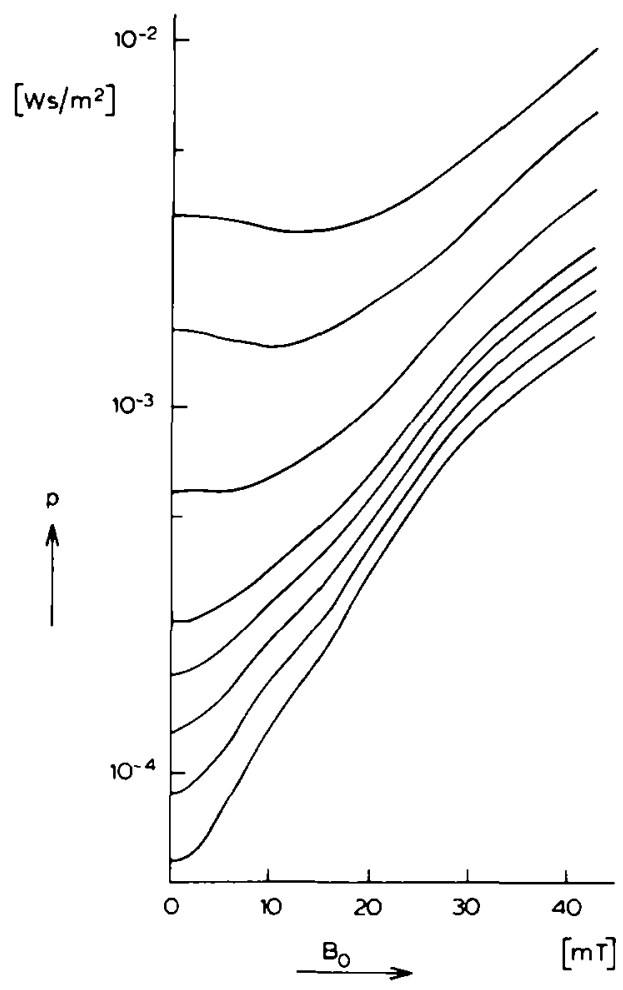

Fig. 10. Calculated a.c. losses as a function of static field in the case of realistic $B_{\text {en }}\left(B_{\mathrm{a}}\right)$ and $B_{\text {ex }}\left(B_{\mathrm{a}}\right)$ relations for various amplitudes $\left(b_{0}=23,24,25,26,27,30,35,40 \mathrm{mT}\right.$; $\left.j_{\mathrm{c}}(0)=0.212 \times 10^{6} \mathrm{~A} \mathrm{~cm}^{-2} ; B_{0}=130 \mathrm{mT}\right)$.

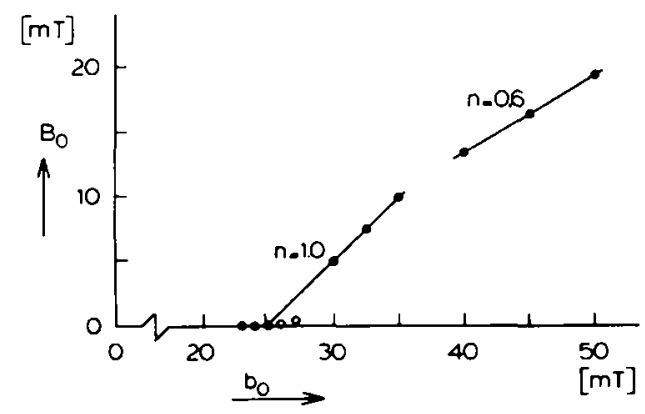

Fig. 11. The static field at which the minimum occurs as a function of $b_{0}$ in the case of realistic $B_{\mathrm{en}}\left(\boldsymbol{B}_{\mathrm{a}}\right)$ and $\boldsymbol{B}_{\mathrm{ex}}\left(\boldsymbol{B}_{\mathrm{a}}\right)$ relations (see fig. 10).

however, differs from the experimental value $\boldsymbol{n}=\mathbf{0 . 8 6}$.

(2) the straight line with slope $n=1$ crosses the axis $B_{\min }=0$ at $b_{0}=25 \mathrm{mT}$. This value, which is in agreement with the experimental value, is much larger than the amplitude of first penetration $\left(b_{0}=14 \mathrm{mT}\right)$.
(3) also for amplitudes slightly exceeding $b_{0}$ $=25 \mathrm{mT}$, the minimum occurs at $B_{0}=0 \mathrm{mT}$.

It may be concluded from the foregoing that the calculated results involving $B_{\text {en }}\left(B_{\mathrm{a}}\right)$ and $B_{\text {ex }}\left(B_{\mathrm{a}}\right)$ curves determined from experiments agree excellently with experimental results on a.c. losses. Only one deviation can be observed: the slopes in the low amplitude region have different values. A possible explanation for this deviation is that the actual $j_{\mathrm{c}}(B)$ relation does not fully obey relation (3).

\section{Conclusions}

In this paper a numerical technique has been presented which allows the calculation of flux distributions, induced voltage waveforms, hysteresis loops and a.c. losses under quasi-static conditions for arbitrary $j_{\mathrm{c}}(B, x), B_{\mathrm{en}}\left(B_{\mathrm{a}}\right)$ and $B_{\mathrm{ex}}\left(B_{\mathrm{a}}\right)$ relations. In addition to earlier publications $[5,6]$ both the finite thickness of the sample and the influence of both surface and bulk currents have been taken into account. Experimental $B_{\text {en }}$ and $B_{\text {ex }}$ curves have also been included. The calculations are based on the Critical State Model. Some calculated induced voltage waveforms and hysteresis loops have been presented; a comparison with experimental results will be given in part II [10].

The calculated a.c. losses exhibit a minimum when a static field $B_{0}$ is superposed on the a.c. field. It has been found that most of the deviations between experimental values and calculated results presented earlier [6] , can be explained by taking into account the finite thickness of the slab and experimental $B_{\text {en }}\left(B_{\mathrm{a}}\right)$ and $B_{\text {ex }}\left(B_{\mathrm{a}}\right)$ relation. The excellent agreement in the occurrence of the minima which has been obtained with experimental $B_{\text {en }}$ and $B_{\text {ex }}$ curves, is an indication for the correctness of these curves. The influence of a position dependent $j_{\mathrm{c}}$ on the a.c. losses has been found to be of minor importance.

\section{Acknowledgement}

The authors would like to acknowledge M. Ciszek and G. Kozlowski for their contribution to the discussions and for placing at our disposal the experimental results on a.c. losses. We also thank J. L. de Reuver for the determination of $B_{\mathrm{en}}\left(B_{\mathrm{a}}\right)$ and $B_{\mathrm{ex}}$ $\left(B_{\mathrm{a}}\right)$. 


\section{References}

[1] A. M. Campbell and J. E. Evetts, Adv. Phys, 21 (1972) 199.

[2] L. J. M. van de Klundert, E. A. Gijsbertse and H. P. van der Braak, Physica 94B (1978) 41.

[3] E. A. Gijsbertse, M. Caspari and L. J. M. van de Klundert, Cryogenics, accepted for publication.

[4] E. A. Gijsbertse, Thesis, Enschede (October 1980).

[5] D. G. Walmsley, J. Appl. Phys. 43 (1972) 615.

[6] J. R. Clem, J. Appl. Phys. 50 (1979) 3518.

[7] J. D. Thompson, M. P. Maley and J. R. Clem, J. Appl. Phys. 50 (1979) 3531.

[8] M. Ciszek, G. Kozlowski and P. Tekiel, Phys. Stat. Sol., to be published.
[9] M. Ciszek, G. Kozlowski, P. Tekiel, E, A. Gijsbertse and L. J. M. van de Klundert, Phys. Lett. 77A (1980) 271.

[10] E. A. Gijsbertse, J. L. de Reuver and L. J. M. van de Klundert, to be published.

[11] Y. B. Kim, C. F. Hempstead and A. R. Strnad, Phys. Rev. 129 (1963) 528.

[12] E. A. Gijsbertse and L. J. M. van de Klundert, Physica $103 B$ (1981) 315 .

[13] J. R. Clem, Proc. LT 13, vol. 3 (Plenum Press, New York, 1974) p. 102.

[14] E. A. Gijsbertse and L. J. M. van de Klundert, Phys. Lett., submitted. 